\title{
A Faster R CNN-Based Real-Time QRS Detector
}

\author{
Hao Yang ${ }^{1}$, Maolin Huang ${ }^{1}$, Zhipeng $\mathrm{Cai}^{2}$, Yingjia Yao ${ }^{3}$, Chengyu Liu ${ }^{2}$ \\ ${ }^{1}$ Lenovo Research Institute, Shenzhen, China \\ ${ }^{2}$ School of Instrument Science and Engineering, Southeast University, Nanjing, China \\ ${ }^{3}$ Lenovo Research Institute, Beijing, China
}

\begin{abstract}
Accurate QRS location keeps challenging in dynamic electrocardiograms (ECGs). This study addressed this issue and developed a novel faster $R$ convolutional neural network (CNN) model-based real-time QRS detection algorithm. Firstly, ECGs were segmented into 10-s length episodes, and each episode was transformed into a 2-D image with a pixel size of $200 \times 200$ (VOC2007 format). Labelled QRS location information was used to generate the QRS bounding boxes. A faster $R C N N$ model was constructed. Candidates of $Q R S$ bounding boxes were extracted by the region proposal networks (RPN). Then, the boxes with small probabilities were excluded according to the rules of probability distribution and QRS location relationship. Finally, locations of QRS complexes were determined based on the geometric features and threshold rule. The proposed algorithm was trained on the MIT/BIH arrhythmia database and verified on the 24-h wearable ECGs. Five-fold cross validation on 24-h wearable ECG recordings from 20 subjects generated a sensitivity of $98.76 \%$, a positive predictivity of $98.52 \%$ and an accuracy of $97.32 \%$ compared to the manual annotations. In addition, the cost time of the new algorithm for processing a 10-s ECG episode was less than $20 \mathrm{~ms}$ under the experiments of $C P U$ i7-2600 $3.40 \mathrm{GHz}, 8 \mathrm{~GB}$ RAM, tesla M60 GPU and 16 GB graphics memory.
\end{abstract}

\section{Introduction}

Electrocardiogram (ECG) is a comprehensive manifestation of cardiac electrophysiological activity, providing important information about the state of cardiac function [1]. Therefore, automatic diagnosis of static or long-term dynamic ECG is essential for doctors and patients. The accurate QRS recognition algorithm is the basis for automatic ECG analysis, which is important for the prevention and diagnosis of heart diseases [2, 3]. However, QRS detection in dynamic ECG, especially in wearable ECG, has encountered significant challenges due to motion disturbances, ECG electrode contact and myoelectric noise [4]. QRS detectors must be able to correctly detect different forms of QRS with large amounts of noise. However, the anti-noise and anti-interference ability of existing QRS detection algorithms is not strong enough $[5,6]$.

In general, in order to accurately identify the QRS complex, it is necessary to effectively pre-process the original ECG, which can suppress power frequency interference, myoelectric interference, respiratory interference and baseline noise, and help identify QRS complexes [7], such as Pan-Tompkins algorithm [8]. Kim and Shin [9] developed spatiotemporal characteristic-based detector, where the maximum energy level within the 5-25 $\mathrm{Hz}$ frequency band was accepted to belong to QRS complexes. Christov's QRS detector [10] used the information from more than one simultaneously recorded channels, with three special threshold rules: adaptive slewrate, correction depending on the presence of highfrequency noise and low amplitude QRS complexes [11].

Although various QRS detectors have been developed, their anti-interference and anti-distortion abilities still need to be improved, especially when considering to combine some machine learning methods. This paper proposes a faster R convolutional neural network (CNN) model-based real-time QRS detector to address this problem.

\section{Methods}

\subsection{Data}

MIT/BIH arrhythmia database was used as training dataset. Wearable ECG data collected from a portable ECG device was used as test dataset, which included the 24-h ECGs from 20 patients with different arrythmia types. All collected wearable ECG data were labeled by two independent cardiologists and arbitrated by a third.

\subsection{Generation of $Q R S$ region of interest}

To apply the fast R CNN model, ECG signal has to be transformed into 2-D images. Firstly, ECG signals were segmented into 10-s segments. Secondly, in order to remove baseline drift and power frequency interference, a $0.5-40 \mathrm{~Hz}$ band pass filter was applied to the 10-s ECG segments. Thirdly, normalization was applied to normalize 
the ECG amplitude within 0 and 1, which can eliminate the adverse effects caused by the noises. Then, 1-D data was transformed into $200 \times 200$ pictures through the matplotlib tools in Python. Finally, since the width of the QRS is usually about $120 \mathrm{~ms}$, the rectangular range of the QRS complex was limited $100 \mathrm{~ms}$ forward and $100 \mathrm{~ms}$ backward at the position of $\mathrm{R}$ peak. Fig. 1 shows the process of generating ECG image datasets. Fig. 2 demonstrates an example of QRS bounding boxes in the ECG image.

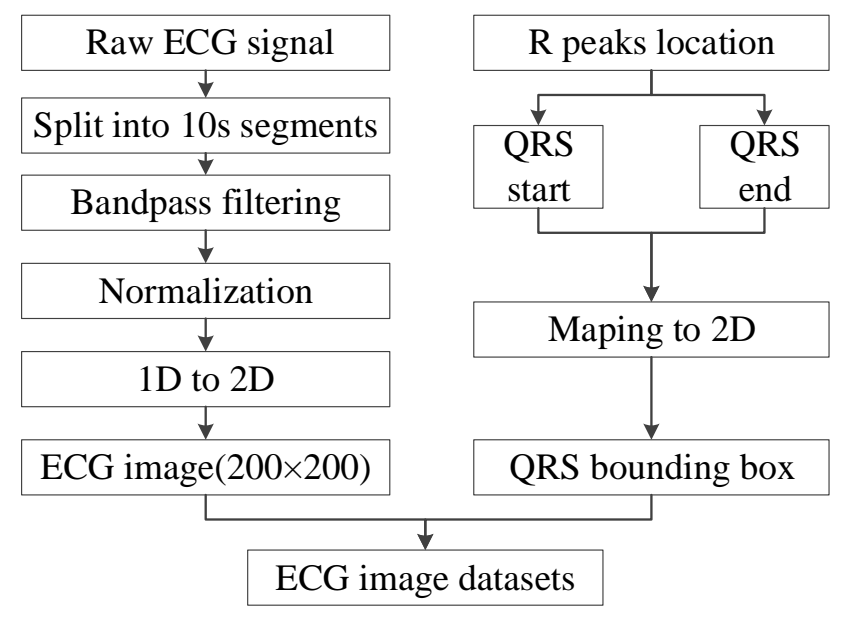

Figure 1. Generation of ECG samples for the faster R CNN-based QRS detector.

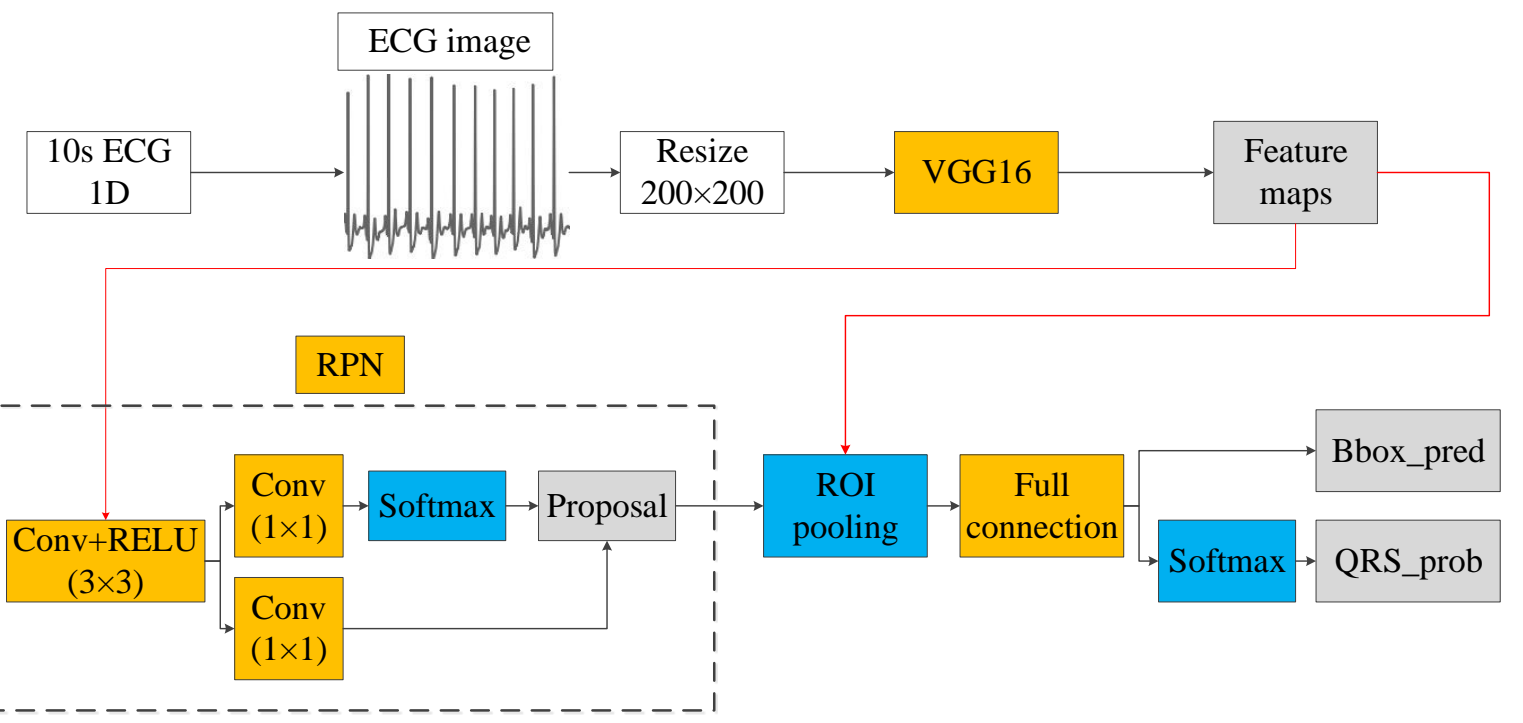

Figure 3. Model structure of the proposed faster R CNN-based QRS detector. VGG16 is used for feature extraction, RPN layer is used to select candidate targets, ROI pooling layer maps the proposals to the feature map.

\subsection{Parameter setting}

Before training, we need to pre-set several parameters. The loss function of fast R-CNN is set as:

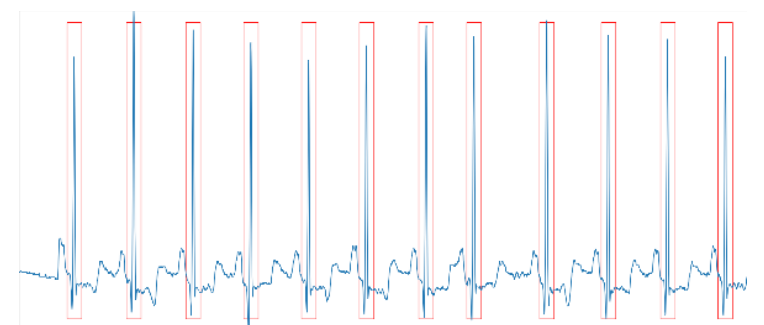

Figure 2. An example of QRS bounding boxes (red rectangles) within a $10-\mathrm{s}$ ECG segment.

\subsection{Faster R CNN Model}

As shown in Fig. 3, a 10-s ECG 2-D image $(200 \times 200)$ was first put into the feature extraction network of VGG16, which included 13 convolution layers, 13 rectified linear units (RELU) layers and 4 pooling layers. Then the feature maps were put into a regional proposal networks (RPN) network [12], which included two branches. The top branch obtained foreground (QRS region of interest (ROI)) and background by the softmax function. The low branch calculated bounding box regression offset to get accurate proposals [13]. ROI pooling layer mapped the proposals to the feature map and resized these regions into same size sections by max pooling operation [12]. The classifier layers got the precise locations of QRS complexes by the fully connected layers and softmax function. 
$t_{i}$, representing the actual offset of anchor relative to ground truth, $p_{i}$ is the probability of anchor prediction, and $p_{i}^{*}$ represents the ground truth.

There are two parts in formula (1), the first part is the classification loss and the second part is bounding box regression loss. Classification loss is cross-entropy loss function, which means calculating logarithmic loss for each anchor and dividing the sum by the total number of anchors $\frac{1}{N_{c l s}}$. Usually, $f(x)=x^{2}$ is chosen in Bounding box regression. However, this loss function will be very high if large errors are encountered [14]. Therefore, slightly flat absolute loss function, i.e., $f(x)=|x|$, is applied herein, which increases linearly with the error rather than squarely. The derivative of this function does not exist at 0 , which has a bad influence on the convergence of functions. Piecewise function is introduced to make it smoother by using square function near 0 , named smooth L1 loss function. In this study, the smooth L1 loss function is defined by

$$
\operatorname{smooth}_{L 1}(x)=\left\{\begin{array}{l}
0.5 x^{2} \times \frac{1}{\sigma^{2}}, \text { if }|x|<\frac{1}{\sigma^{2}} \\
|x|-0.5, \text { otherwise }
\end{array}\right.
$$

where $x$ represents the difference between the actual offset of the border and the predicted offset $x=t_{i}-t_{i}^{*}$ and $\sigma=$ 3 . Learning rate was set 0.001 , Gradient descent method with momentum was chosen as optimization method.

\subsection{QRS location with the model output}

ROI of QRS information can be obtained by the proposed fast R-CNN Model. As is shown in Fig. 2, the R peaks are located inside the red rectangles. The slope of QRS is the most obvious feature [15]. In order to extract the location of QRS more accurately, the bilateral slope method was used to precisely locate the position of $\mathrm{R}$ peaks:

$$
\begin{aligned}
& \text { slop } L_{i}=e c g_{i}-e c g_{i-4}, \\
& \operatorname{slop} R_{i}=e c g_{i}-e c g_{i+4},
\end{aligned}
$$

where $\operatorname{slop}_{i}$ and $\operatorname{slop} R_{i}$ indicate the left and right slopes, respectively, $e c g_{i}$ represents the ECG amplitude.

\section{Results and discussion}

Five-fold cross validation on the MIT/BIH arrhythmia database achieved a sensitivity $(\mathrm{Se})$ of $99.24 \%$ and a positive predictivity $(+P)$ of $99.90 \%$, resulting in an accuracy (Acc) of $99.14 \%$, as shown in table 1 . When tested on the twenty 24-h wearable ECG recordings, the algorithm generated a $\mathrm{Se}$ of $98.76 \%$, a $+P$ of $98.52 \%$ and an Acc of $97.32 \%$ compared to the manual annotations.

From Tables 1 and 2, we can see that the proposed fast R-CNN algorithm can well perform the QRS detection task. Although trained on the MIT/BIH arrhythmia database, the QRS detector can still have a good performance on wearable ECG data, which shows a strong generalization ability.
Table 1. Five-fold cross validation results on the MIT/BIH arrythmia database.

\begin{tabular}{lcccccc}
\hline Fold & TP & FP & FN & $S e(\%)$ & $+P(\%)$ & $A c c(\%)$ \\
\hline 1 & 19059 & 64 & 78 & 99.59 & 99.67 & 99.26 \\
2 & 18916 & 7 & 131 & 99.31 & 99.96 & 99.28 \\
3 & 21542 & 21 & 257 & 98.82 & 99.9 & 98.73 \\
4 & 26449 & 2 & 195 & 99.27 & 99.99 & 99.26 \\
5 & 20823 & 10 & 164 & 99.22 & 99.95 & 99.17 \\
\hline Mean & $/$ & $/$ & $/$ & 99.24 & 99.90 & 99.14 \\
SD & $/$ & $/$ & $/$ & 0.08 & 0.02 & 0.05 \\
\hline
\end{tabular}

\begin{tabular}{|c|c|c|c|c|c|c|}
\hline \#subject & $\mathrm{TP}$ & FP & $\mathrm{FN}$ & $\begin{array}{l}\mathrm{Se} \\
(\%)\end{array}$ & $\begin{array}{l}+P \\
(\%)\end{array}$ & $\begin{array}{l}A c c \\
(\%)\end{array}$ \\
\hline 1 & 132957 & 1229 & 624 & 99.53 & 99.08 & 98.63 \\
\hline 2 & 100345 & 392 & 542 & 99.46 & 99.61 & 99.08 \\
\hline 3 & 73028 & 1664 & 312 & 99.57 & 97.77 & 97.37 \\
\hline 4 & 98905 & 1383 & 906 & 99.09 & 98.62 & 97.74 \\
\hline 5 & 133725 & 793 & 3907 & 97.16 & 99.41 & 96.60 \\
\hline 6 & 97811 & 1417 & 2492 & 97.52 & 98.57 & 96.16 \\
\hline 7 & 115364 & 58 & 540 & 99.53 & 99.95 & 99.48 \\
\hline 8 & 67346 & 1698 & 958 & 98.60 & 97.54 & 96.21 \\
\hline 9 & 77003 & 1097 & 625 & 99.19 & 98.60 & 97.81 \\
\hline 10 & 107291 & 1624 & 1288 & 98.81 & 98.51 & 97.36 \\
\hline 11 & 110539 & 4653 & 1943 & 98.27 & 95.96 & 94.37 \\
\hline 12 & 79257 & 2061 & 488 & 99.39 & 97.47 & 96.88 \\
\hline 13 & 97228 & 255 & 217 & 99.78 & 99.74 & 99.52 \\
\hline 14 & 108798 & 448 & 992 & 99.10 & 99.59 & 98.69 \\
\hline 15 & 97133 & 1356 & 1682 & 98.30 & 98.62 & 96.97 \\
\hline 16 & 71810 & 3213 & 1497 & 97.96 & 95.72 & 93.84 \\
\hline 17 & 86631 & 677 & 689 & 99.21 & 99.22 & 98.45 \\
\hline 18 & 70608 & 1308 & 1118 & 98.44 & 98.18 & 96.68 \\
\hline 19 & 114731 & 893 & 2164 & 98.15 & 99.23 & 97.40 \\
\hline 20 & 110933 & 1047 & 2140 & 98.11 & 99.07 & 97.21 \\
\hline Mean & I & I & I & 98.76 & 98.52 & 97.32 \\
\hline SD & I & I & I & 0.55 & 1.34 & 2.24 \\
\hline
\end{tabular}

Table 2. Results of the proposed QRS detector on the twenty 24-h wearable ECG recordings.

We also tested the calculation cost. The cost time of the new algorithm for processing a 10-s ECG episode was less than $20 \mathrm{~ms}$ under the experiments of CPU i7-2600 3.40 GHz, 8 GB RAM, tesla M60 GPU and 16 GB graphics memory. The new QRS detector should be carried out on GPU, and thus the resource consumption is large. It still needs to be optimized in resource consumption to facilitate the practical use in the future. 
Traditional QRS detection methods are usually affected by noise and QRS shape, which is hard to improve $+P$ and $\mathrm{Se}$ simultaneously. In the contrary, the new QRS detector can deal with the QRS detection under different noise situation, generating an increase of $+P$ and $S e$ simultaneously. In addition, the proposed method can not only used for QRS detection, but also has potential for other feature identification, such as $\mathrm{T}$ waves, $\mathrm{P}$ waves.

\section{Conclusion}

This study presents an effective QRS detection method based on Fast R CNN model, which generated a sensitivity of $98.76 \%$, a positive predictivity of $98.52 \%$ and an accuracy of $97.32 \%$ on the actually collected twenty $24-\mathrm{h}$ wearable ECG recordings. It is only a polit study, and more work need to further improve the issue of resource consumption.

\section{Acknowledgement}

The study was partly supported by the National Natural Science Foundation of China (81871444). The authors thank the support from the Southeast-Lenovo Wearable Heart-Sleep-Emotion Intelligent monitoring Lab.

\section{References}

[1] X. Xu and Y. Liu, "ECG QRS complex detection using slope vector waveform (SVW) algorithm," in The 26th Annual International Conference of the IEEE Engineering in Medicine and Biology Society, pp. 3597-3600, 2004.

[2] S. Mitra, M. Mitra and B. B. Chaudhuri, "Pattern defined heuristic rules and directional histogram based online ECG parameter extraction," Measurement, vol. 42, pp. 150-156, 2009.

[3] F. Zhang and Y. Lian, "QRS detection based on multiscale mathematical morphology for wearable ECG devices in body area networks," IEEE Transactions on Biomedical Circuits and Systems, vol. 3, pp. 220-228, 2009.

[4] C. Liu, X. Zhang, L. Zhao, F. Liu, X. Chen, Y. Yao, and J. $\mathrm{Li}$, "Signal quality assessment and lightweight QRS detection for wearable ECG SmartVest system," IEEE Internet of Things Journal, vol. 6, pp. 1363-1374, 2018.

[5] F. Liu, C. Liu, X. Jiang, Z. Zhang, Y. Zhang, J. Li, and S.
Wei, "Performance analysis of ten common QRS detectors on different ECG application cases," Journal of Healthcare Engineering, vol. 2018, 2018.

[6] F. Liu, S. Wei, Y. Li, X. Jiang, Z. Zhang, L. Zhang, and C. Liu, "The accuracy on the common Pan-Tompkins based QRS detection methods through low-quality electrocardiogram database," Journal of Medical Imaging and Health Informatics, vol. 7, pp. 1039-1043, 2017.

[7] Y. Yeh and W. Wang, "QRS complexes detection for ECG signal: The Difference Operation Method," Computer Methods and Programs in Biomedicine, vol. 91, pp. 245254, 2008.

[8] J. Pan and W. J. Tompkins, "A real-time QRS detection algorithm," IEEE Transaction on Biomedicine and Engineering, vol. 32, pp. 230-236, 1985.

[9] D. S. Kim, J. E. Kim, S. E. Kwak, K. C. Choi, D. W. Kim, O. S. Kwon, S. Y. Choi, and T. C. Kang, "Spatiotemporal characteristics of astroglial death in the rat hippocampo entorhinal complex following pilocarpine - induced status epilepticus," Journal of Comparative Neurology, vol. 511, pp. 581-598, 2008.

[10] K. Daskalov and I. I. Christov, "Electrocardiogram signal preprocessing for automatic detection of QRS boundaries," Medical Engineering \& Physics, vol. 21, pp. 37-44, 1999.

[11] Christov and T. V. Stoyanov, "Steep slope method for real time QRS detection," Electrotechnics \& Electronics E+ E, vol. 1, pp. 13-17, 2002.

[12] Z. Cai, Q. Fan, R. S. Feris, and N. Vasconcelos, "A unified multi-scale deep convolutional neural network for fast object detection," in European Conference on Computer Vision, 2016, pp. 354-370.

[13] S. Ren, K. He, R. Girshick, and J. Sun, "Faster r-cnn: Towards real-time object detection with region proposal networks," in Advances in Neural Information Processing Systems, 2015, pp. 91-99.

[14] P. Bühlmann and B. Yu, "Boosting with the L 2 loss: regression and classification," Journal of the American Statistical Association, vol. 98, pp. 324-339, 2003.

[15] D. Shishir, J. G. Quirk and P. Djuric, "Apparatus and method for feature extraction and classification of fetal heart rate,": Google Patents, 2014.

Address for correspondence.

Chengyu Liu, $\mathrm{PhD}$

School of Instrument Science and Engineering

Southeast University, Nanjing, China

E-mail: chengyu@seu.edu.cn 